\title{
Existence of Positive Solutions for Second-Order Third-Point Semipositive BVP
}

\author{
Hua Su $(\mathbb{D}$ and Jinmin Yu \\ School of Mathematics and Quantitative Economics, Shandong University of Finance and Economics, Jinan, \\ Shandong 250014, China \\ Correspondence should be addressed to Hua Su; jnsuhua@163.com
}

Received 28 April 2021; Accepted 21 June 2021; Published 7 July 2021

Academic Editor: Xinguang Zhang

Copyright ( 92021 Hua Su and Jinmin Yu. This is an open access article distributed under the Creative Commons Attribution License, which permits unrestricted use, distribution, and reproduction in any medium, provided the original work is properly cited.

In this paper, we study the existence of positive solutions for the following nonlinear second-order third-point semi-positive BVP. We derive an explicit interval of positive parameters, which for any $l, \mu$ in this interval, the existence of positive solutions to the boundary value problem is guaranteed under the condition that $a(t, x), b(t, x)$ are all superlinear (sublinear), or one is superlinear, the other is sublinear.

\section{Introduction}

In the applied mathematical field, three-point BVP can describe many phenomena. Moshinsky [1] introduced the vibrations of a guy wire with a uniform cross-section and composed of $N$ parts of different densities using a multipoint BVP. Timoshenko [2] also revealed that the theory of elastic stability can be used by the method of a three-point BVP. Il'in and Moviseev [3] were the first to study this aspect. Since then, more general nonlinear BVP have been studied by several authors [4-25].

In their paper [7], Ma and Wang obtained the existence of positive solutions for a three-point BVP by Krasnoselskii's fixed theorem:

$$
\begin{cases}u^{\prime \prime}(t)+a(t) u^{\prime}(t)+b(t) u(t)+h(t) f(u)=0, & 0 \leq t \leq 1, \\ u(0)=0, & u(1)=\alpha u(\eta),\end{cases}
$$

where $\alpha$ is a positive constant, $0<\eta<1, a(t) \in C\left([0,1], \mathbf{R}^{+}\right)$, $b(t) \in C\left([0,1], \mathbf{R}^{-}\right), f \in C\left(\mathbf{R}^{+}, \mathbf{R}^{+}\right), h \in C\left([0,1], \mathbf{R}^{+}\right)$and there exists $x_{0} \in(0,+\infty)$ such that $h\left(x_{0}\right)>0$.
In our paper, we study the existence of positive solutions of second-order third-point semipositive BVP:

$$
\begin{cases}(L x)(t)+\lambda a(t, x)+\mu b(t, x), & 0 \leq t \leq 1, \\ x(0)=0, & x(1)=\alpha x(\xi),\end{cases}
$$

where $(L u)(t)=u^{\prime \prime}(t)+f(t) u^{\prime}(t)+g(t) u(t), \lambda, \mu$ are positive parameters, $0<\xi<1, f(t) \in C[0,1]$, and $g(t) \in C([0,1]$, $(-\infty, 0))$. And our paper also allows that $a(t, x), b(t, x)$ are both semipositive and lower unbounded.

Our main tool is the following fixed point index theory.

Theorem 1 [4]. We suppose that $K \subset E$ is a cone in $E$, in which $E$ is a real Banach space, the open bounded set $\Omega_{1}, \Omega_{2}$ is in $E$, $\theta \in \Omega_{1}, \quad \bar{\Omega}_{1} \subset \Omega_{2}$, and $T: K \cap\left(\bar{\Omega}_{2} \backslash \Omega_{1}\right) \longrightarrow K$. Suppose operator $T$ can be completely continuous and satisfies one of the following conditions:

(i) $\|T x\| \leq\|x\|, \quad \forall x \in K \cap \partial \Omega_{1} ; \quad\|T x\| \geq\|x\|, \quad \forall x \in K \cap$ $\partial \Omega_{2}$

(ii) $\|T x\| \geq\|x\|, \quad \forall x \in K \cap \partial \Omega_{1} ; \quad\|T x\| \leq\|x\|, \quad \forall x \in K \cap$ $\partial \Omega_{2}$ 
Then, operator $T$ has at least one fixed point $x^{*}$ in $K$ $\cap\left(\bar{\Omega}_{2} \backslash \Omega_{1}\right)$.

Theorem 2 [4]. We suppose that $P \subset E$ is a cone in $E$, in which $E$ is a real Banach space, the open bounded set $\Omega_{1}, \Omega_{2}, \Omega_{3}$ is in $E, \theta \in \Omega_{1}, \bar{\Omega}_{1} \subset \Omega_{2}, \bar{\Omega}_{2} \subset \Omega_{3}$, and $T: P \cap \Omega_{3} \longrightarrow$ P. Suppose operator $A$ is completely continuous and satisfies the following conditions:

$$
\begin{array}{ll}
\|T x\| \leq\|x\|, & \forall x \in P \cap \partial \Omega_{1}, \\
\|T x\| \geq\|x\|, & A x \neq x, \forall x \in P \cap \partial \Omega_{2}, \\
\|T x\| \leq\|x\|, & \forall x \in P \cap \partial \Omega_{3} .
\end{array}
$$

Then, operator $T$ has at least two fixed points $x^{*}$ and $x^{* *}$ in $P \cap\left(\bar{\Omega}_{3} / \Omega_{1}\right)$, and $x^{*} \in P \cap\left(\Omega_{2} / \Omega_{1}\right)$ and $x^{* *} \in P \cap\left(\bar{\Omega}_{3} / \bar{\Omega}_{2}\right)$.

\section{Preliminaries and Lemmas}

We set a Banach space $E=C([0,1],(-\infty,+\infty))$ with norm $\|x\|=\max _{t \in I}|x(t)|$. We know of the following lemmas from Ref. [6].

Lemma 3. Setting $\xi_{1}(t)$ as the positive solution of the equation, we have:

$$
\begin{cases}\left(L \xi_{1}\right)(t)=0, & 0 \leq t \leq 1, \\ \xi_{1}(0)=0, & \xi_{1}(1)=1 .\end{cases}
$$

Then, $\xi_{1}(t) \in[0,1]$ is strictly increasing on $[0,1]$, and $\xi_{1^{\prime}}$ (0) $>0$.

Lemma 4. Setting $\xi_{2}(t)$ as the positive solution of the equation, we have:

$$
\begin{cases}\left(L \xi_{2}\right)(t)=0, & 0 \leq t \leq 1, \\ \xi_{2}(0)=1, & \xi_{2}(1)=0 .\end{cases}
$$

Then, $\xi_{2}(t) \in[0,1]$ is strictly decreasing on $[0,1]$.

From Lemma 3 and Lemma 4, we know that $0<\xi_{1}(t)<1$, $0<\xi_{2}(t)<1$. In the rest of our paper, the following condition is used:

(C1) $0<\alpha \xi_{1}(\eta)<1$, where $\xi_{1}(t)$ is given by Lemma 3

Throughout this paper, we shall use the following notation:

$$
G(t, s)=\frac{1}{\zeta} \begin{cases}\xi_{1}(t) \xi_{2}(s), & 0 \leq t \leq s \leq 1 \\ \xi_{1}(s) \xi_{2}(t), & 0 \leq s \leq t \leq 1\end{cases}
$$

where $\zeta=\xi_{1}^{\prime}(0) \xi_{2}(0)$.
Obviously, from Ref. [6], we can be assured that when (C1) holds, the BVP

$$
\begin{cases}(L x)(t)+y(t)=0, & 0 \leq t \leq 1, \\ x(0)=0, & x(1)=\alpha u(\xi),\end{cases}
$$

is equivalent to the following integral equation:

$$
x(t)=\int_{0}^{1} G(t, s) e(s) y(s) d s+\frac{\alpha \xi_{1}(t)}{1-\alpha \xi_{1}(\xi)} \int_{0}^{1} G(\xi, s) e(s) y(s) d s,
$$

where $e(t)=\exp \left(\int_{0}^{t} f(s) d s\right)$.

Set $z(t)=\min \left(\left(\xi_{1}(t) /\left\|\xi_{1}\right\|\right),\left(\xi_{2}(t) /\left\|\xi_{2}\right\|\right)\right)$. From (6), for $t \in[0,1]$, we know that

$$
z(t) G(s, s) \leq G(t, s) \leq G(s, s) .
$$

We present some other lemmas that are important to our main results.

Lemma 5 [7]. Assume that for any $y \in C([0,1],(0,+\infty)), x(t)$ is the solution of the following $B V P$ :

$$
\begin{cases}(L x)(t)+y(t)=0, & 0<t<1, \\ x(0)=0, & x(1)=\alpha x(\xi) .\end{cases}
$$

Then, we have

$$
x(t) \geq z(t)\|x\|, t \in[0,1] .
$$

Lemma 6. Assume that $\bar{w}$ is a solution of the following BVP:

$$
\begin{cases}(L x)(t)=-B(t), & 0<t<1, \\ x(0)=0, & x(1)=\alpha x(\xi),\end{cases}
$$

where $B \in C(0,1), M>0$. Then, there exists constant $M>0$ and satisfies

$$
\bar{w}(t) \leq M\|B\| z(t), \quad t \in[0,1] .
$$

Proof. For $t \in[0,1]$, we can have

$$
\bar{w}(t)=\int_{0}^{1} G(t, s) e(s) B(s) d s+\frac{\alpha \xi_{1}(t)}{1-\alpha \xi_{1}(\xi)} \int_{0}^{1} G(\xi, s) e(s) B(s) d s .
$$


Obviously, for $t \in[0,1]$, we have

$$
\begin{aligned}
\int_{0}^{1} G(t, s) e(s) B(s) d s \\
=\frac{1}{\zeta}\left[\int_{0}^{t} \xi_{1}(s) \xi_{2}(t) e(s) B(s) d s+\int_{t}^{1} \xi_{1}(t) \xi_{2}(s) e(s) M(s) d s\right] \\
\leq \frac{p(1)}{\zeta}\left[\xi_{1}(t) \xi_{2}(t) \int_{0}^{t} B(s) d s+\xi_{1}(t) \xi_{2}(t) \int_{t}^{1} B(s) d s\right] \\
=\frac{e(1)\left\|\xi_{1}\right\|\left\|\xi_{2}\right\|}{\zeta}\left[\frac{\xi_{1}(t)}{\left\|\xi_{1}\right\|} \frac{\xi_{2}(t)}{\left\|\xi_{2}\right\|} \int_{0}^{t} M(s) d s\right. \\
\left.\quad+\frac{\xi_{1}(t)}{\left\|\xi_{1}\right\|} \frac{\xi_{2}(t)}{\left\|\xi_{2}\right\|} \int_{t}^{1} B(s) d s\right] \\
\leq \frac{e(1)\left\|\xi_{1}\right\|\left\|\xi_{2}\right\|}{\zeta} z(t) \int_{0}^{1} B(s) d s \leq M_{1} z(t)\|B\|
\end{aligned}
$$

where $M_{1}=\left(e(1)\left\|\xi_{1}\right\|\left\|\xi_{2}\right\|\right) / \zeta$.

By the same method, we can know that

$$
\frac{\alpha \xi_{1}(t)}{1-\alpha \xi_{1}(\xi)} \int_{0}^{1} G(\xi, s) e(s) B(s) d s \leq M_{2} z(t)\|B\|,
$$

where $M_{2}=\left(\alpha e(1)\left\|\xi_{1}\right\|\left\|\xi_{2}\right\|\right) /\left(1-\alpha \xi_{1}(\xi)\right)$.

So, by choosing constant $M \geq M_{1}+M_{2}$, we have

$$
\bar{w}(t) \leq M\|B\| z(t), \quad 0 \leq t \leq 1
$$

Lemma 7 [7]. Let $0 \leq \lim _{x \infty}^{-}(b(t, x) / x) \leq L_{2}, t \in[0,1]$. Define the following function:

$$
G(\tau)=\max _{0 \leq t \leq 1,0 \leq x \leq \tau} b(t, \tau)
$$

Then

(i) $G$ is a nondecreasing function for $\tau$

(ii) $0 \leq \lim _{\rho \infty}^{-}(G(\tau) / \tau) \leq K_{2}$

For g assumptions:

(C2) $a(t, x), b(t, x) \in C,([0,1] \times[0,+\infty) R)$

From (C2), there exists a function $B(t) \in C[0,1], B(t)>0$, which satisfies

$$
\begin{aligned}
a(t, x) & \geq-B(t), \\
b(t, x) & \geq-B(t), \\
\forall t & \in(0,1), x \geq 0,
\end{aligned}
$$

where $M\|B\|<1$. M is given by Lemma 6 .
(C3) $B_{1} \leq a_{\infty}^{-} \leq \infty, B_{2} \leq b_{\infty}^{-} \leq \infty$
(C4) $0 \leq a_{\infty}^{+} \leq b_{1}, 0 \leq b_{\infty}^{+} \leq b_{2}$
(C5) $K_{1} \leq a_{\infty}^{-} \leq \infty, 0 \leq b_{\infty}^{+} \leq K_{2}$

where

$$
\begin{aligned}
& \min \left(B_{1}, B_{2}\right) \geq 2\left((\lambda+\mu) \min _{0 \leq t \leq 1} \int_{0}^{1} G(t, s) e(s) z(s) d s\right)^{-1}, \\
& b_{1}+b_{2} \leq\left((\lambda+\mu) p(1)\left[\int_{0}^{1} G(s, s) d s+\frac{\alpha \xi_{1}(1)}{1-\alpha \xi_{1}(\xi)} \int_{0}^{1} G(\xi, s) d s\right]\right)^{-1}, \\
& K_{1} \geq 2\left(\lambda \min _{0 \leq t \leq 1} \int_{0}^{1} G(t, s) e(s) z(s) d s\right)^{-1}, \\
& a_{\infty}^{-}=\underline{\lim }_{x \longrightarrow \infty} \frac{a(t, x)}{x}, \\
& a_{\infty}^{+}=\lim _{x \rightarrow \infty} \frac{a(t, x)}{x}, \\
& b_{\infty}^{-}=\underline{\lim }_{x \longrightarrow \infty} \frac{b(t, x)}{x}, \\
& b_{\infty}^{+}=\lim _{x \longrightarrow \infty} \frac{b(t, x)}{x} . \\
& \bar{H}(t, x)= \begin{cases}H(t, x), & x \geq 0, \\
F(t, 0), & x<0,\end{cases} \\
& \bar{Y}(t, x)=\begin{array}{ll}
Y(t, x), & x \geq 0, \\
G(t, 0), & x<0,
\end{array}
\end{aligned}
$$

where $H(t, x)=a(t, x)+B(t), Y(t, x)=b(t, x)+B(t)$.

For any $l>0$, we set

$$
\begin{aligned}
H_{l} & =\max _{0 \leq t \leq 1,0 \leq x \leq l} \bar{H}(t, x), \\
Y_{l} & =\max _{0 \leq t \leq l, 0 \leq x \leq l} \bar{Y}(t, x) .
\end{aligned}
$$

From Lemma 6, letting $w(t)=\bar{w}(t)$, then $x(t)$ is the positive solution of problem (2) if and only if $\tilde{x}(t)=x(t)+w(t)$ is the solution of the following problem:

$$
\left\{\begin{array}{l}
(L x)(t)+\lambda \bar{H}(t, x-w)+\mu \bar{Y}(t, x-w)=0 \\
x(0)=0, \quad x(1)=\alpha u(\xi)
\end{array}\right.
$$

and $\tilde{x}(t)>w(t), 0<t<1$; here, $\bar{H}, \bar{Y}$ is given by (21).

Defining the cone $P$ in $E$, we have

$$
P=\{x \in E: x(t) \geq\|x\| q(t), \quad t \in[0,1]\} .
$$


Obviously, problem (18) is equivalent to

$$
\begin{aligned}
x(t)= & \int_{0}^{1} Y(t, s) e(s)[\lambda \bar{H}(s, x-w)+\mu \bar{Y}(s, x-w)] d s \\
& +\frac{\alpha \xi_{1}(t)}{1-\alpha \xi_{1}(\xi)} \int_{0}^{1} G(\xi, s) e(s)[\lambda \bar{H}(s, x-w)+\mu \bar{Y}(s, x-w)] d s .
\end{aligned}
$$

Defining the operator $T: E \longrightarrow E$, we have

$$
\begin{aligned}
(T x)(t)= & \int_{0}^{1} G(t, s) e(s)[\lambda \bar{H}(s, x-w)+\mu \bar{Y}(s, x-w)] d s \\
& +\frac{\alpha \xi_{1}(t)}{1-\alpha \xi_{1}(\xi)} \int_{0}^{1} G(\xi, s) e(s) \\
& \cdot[\lambda \bar{H}(s, x-w)+\mu \bar{Y}(s, x-w)] d s .
\end{aligned}
$$

Obviously $T(P) \subset P$ and $T$ is completely continuous.

\section{Our Main Three Results}

Theorem 8. Suppose condition (C1), condition (C2), and condition (C3) hold. Then, for the small number $\lambda, \mu$, problem (2) has at least one positive solution.

Proof. Firstly, we choose sufficiently small $\lambda, \mu$ which satisfies the following:

$\lambda+\mu<\left(\left[H_{1}+Y_{1}\right] p(1)\left[\int_{0}^{1} G(s, s) d s+\frac{\alpha \xi_{1}(1)}{1-\alpha \xi_{1}(\xi)} \int_{0}^{1} G(\xi, s) d s\right]\right)^{-1}$.

Letting $\Omega_{1}=\{x \in E:\|x\|<1\}$, for any $x \in P \cap \partial \Omega_{1}, t \in$ $[0,1]$, by the definition of operator $T$, we have

$$
\begin{aligned}
(T x)(t) \leq & \int_{0}^{1} G(s, s)\left[\lambda H_{1}+\mu Y_{1}\right] d s+\frac{\alpha \xi_{1}(t)}{1-\alpha \xi_{1}(\xi)} \\
& \cdot \int_{0}^{1} G(\xi, s) e(s)\left[\lambda H_{1}+\mu Y_{1}\right] d s \\
\leq & (\lambda+\mu)\left[H_{1}+Y_{1}\right] e(1) \\
& \cdot\left[\int_{0}^{1} G(s, s) d s+\frac{\alpha \xi_{1}(1)}{1-\alpha \xi_{1}(\xi)} \int_{0}^{1} G(\xi, s) d s\right]<1=\|x\| .
\end{aligned}
$$

Thus, we have

$$
\|T x\| \leq\|x\|, \quad \forall x \in P \cap \partial \Omega_{1}
$$

Secondly, by (C3), we know that there exists constant $l_{1}>0$ which satisfies

$$
a(t, x) \geq B_{1} x, b(t, x) \geq B_{2} x, \forall x \geq l_{1}, t \in[0,1] .
$$

Letting $r=\max \left\{2 M\|B\|,\left(2 l_{1} / \varepsilon\right), 2\right\}$, then $r>1$. Set $\Omega_{2}=\{x \in E:\|x\|<r\}$, for any $x \in P \cap \partial \Omega_{2}, t \in[0,1]$, we have

$x(t)-w(t) \geq x(t)-M\|B\| z(t) \geq x(t)-\frac{M\|B\|_{1}}{r} x(t) \geq \frac{1}{2} x(t)$.

Therefore, we have $x(t)-w(t) \geq(1 / 2) x(t) \geq(\|x\| / 2) z(t)$ $\geq(\varepsilon r / 2) \geq l_{1}$.

Thus, by the definition of $\bar{H}, \bar{Y}$ and (30), we can have

$$
\begin{aligned}
& \lambda \bar{H}(s, x(s)-w(s))+\mu \bar{Y}(s, x(s)-w(s)) \\
& \quad \geq B_{1} \lambda(x(s)-w(s))+B_{2} \mu(x(s)-w(s)) \\
& \quad \geq \min \left(B_{1}, B_{2}\right)(\lambda+\mu)(x(s)-w(s)) .
\end{aligned}
$$

We have

$$
\begin{aligned}
(T x)(t) & \geq \int_{0}^{1} G(t, s) e(s)[\lambda \bar{H}(s, x-w)+\mu \bar{Y}(s, x-w)] d s \\
& \geq \min _{0 \leq t \leq 1} \int_{0}^{1} G(t, s) \min \left(B_{1}, B_{2}\right)(\lambda+\mu)(x(s)-w(s)) d s \\
& \geq \frac{1}{2}(\lambda+\mu) \min \left(B_{1}, B_{2}\right) \min _{0 \leq t \leq 1} \int_{0}^{1} G(t, s) x(s) d s .
\end{aligned}
$$

Then, by Lemma 5, we have

$\|(T x)(t)\| \geq \frac{1}{2}(\lambda+\mu) \min \left(B_{1}, B_{2}\right) \min _{0 \leq t \leq 1} \int_{0}^{1} G(t, s) e(s) z(s) d s\|x(t)\|$.

Therefore, by the definition of $B_{1}, B_{2}$, we have

$$
\|T x\| \geq\|x\|, \quad \forall x \in K \cap \partial \Omega_{2} .
$$

Then, by (29), (35) and Theorem 1, operator $T$ has at last one fixed point $\tilde{x}(t) \in P \cap\left(\bar{\Omega}_{2} / \Omega_{1}\right)$, i.e., $\tilde{x}(t)$ is the solution of problem (2), and it is easy to know $\|\tilde{x}\| \geq 1$.

Finally, by (C2) and Lemma 3, we have

$$
\tilde{x}(t) \geq\|\tilde{x}\| z(t) \geq z(t)>M\|B\| z(t) \geq \bar{w}(t)=w(t) .
$$

Thus, $x=\tilde{x}-w$ is the positive solution of problem (2).

Theorem 9. We suppose that condition (C1), (C2), and (C4) hold, and the following condition also holds:

(C6) There exist constant $D>0, \rho>0$, and we have

$$
\begin{aligned}
a(t, x) & \geq \rho, \\
b(t, x) & \geq \rho, \\
x & \in[D, \infty), t \in[0,1] .
\end{aligned}
$$


Then, for the small number $\lambda, \mu$, problem (2) has at least one positive solution.

Proof. Firstly, let $r=\max \{2 M\|B\|,(2 D / \varepsilon), 2\}$, and

$$
1=2 r\left(\min _{0 \leq t \leq 1} \int_{0}^{1} Y(t, s) e(s)(\lambda+\mu) \rho d s\right)^{-1} .
$$

Set $\Omega_{1}=\{x \in E:\|x\|<r\}$, for any $x \in P \cap \partial \Omega_{1}, s \in[0,1]$, we have

$$
x(s)-w(s) \geq x(s)-M\|B\| z(s) \geq x(s)-\frac{M\|B\|}{r} x(s) \geq \frac{1}{2} x(s) .
$$

Thus, $x(s)-w(s) \geq(1 / 2) x(s) \geq(\|x\| / 2) z(s) \geq(\varepsilon r / 2) \geq D$. Therefore, by (C6) and the definition of operator $T$, we have

$$
\begin{aligned}
(T x)(t)= & \int_{0}^{1} G(t, s) e(s)[\lambda \bar{H}(s, x-w)+\mu \bar{Y}(s, x-w)] d s \\
& +\frac{\alpha \xi_{1}(t)}{1-\alpha \xi_{1}(\xi)} \int_{0}^{1} G(\xi, s) e(s) \\
& \cdot[\lambda \bar{H}(s, x-w)+\mu \bar{Y}(s, x-w)] d s .
\end{aligned}
$$

For $B(t)>0, t \in(0,1)$, we have

$$
\begin{aligned}
(T x)(t) & \geq \int_{0}^{1} G(t, s) e(s)[\lambda \bar{H}(s, x-w)+\mu \bar{Y}(s, x-w)] d s \\
& \geq \int_{0}^{1} G(t, s) e(s)[\lambda(\rho+B(s))+\mu(\rho+B(s))] d s \\
& \geq \frac{1}{2} \min _{0 \leq t \leq 1} \int_{0}^{1} G(t, s) e(s)(\lambda+\mu) \rho d s=r=\|x\| .
\end{aligned}
$$

We can know that by the above discussion, we have

$$
\|T x\| \geq\|x\|, \forall x \in P \cap \partial \Omega_{1} .
$$

Secondly, by (C4), we can have

$$
\begin{gathered}
0 \leq \lim _{x \rightarrow \infty} \frac{\bar{H}(s, x-w)}{u} \leq b_{1}, \\
0 \leq \lim _{x \rightarrow \infty} \frac{\bar{Y}(s, x-w)}{u} \leq b_{2}, \\
s \in[0,1] .
\end{gathered}
$$

Then, there exists constant $l_{2}>0$ which satisfies

$$
\begin{aligned}
\bar{H}(s, x-w) & \leq b_{1} x, \\
\bar{Y}(s, x-w) & \leq b_{2} x, \\
\forall x & \geq l_{2}, s \in[0,1] .
\end{aligned}
$$

Letting $R=\max \left\{2 l_{2}, 2 r\right\}$, then $r<R$. Set $\Omega_{2}=\{x \in E$ : $\|x\|<R\}$, for any $x \in P \cap \partial \Omega_{2}, t \in[0,1]$, we have

$$
\begin{aligned}
(T x)(t) \leq & \int_{0}^{1} G(s, s)\left[\lambda b_{1} x(s)+\mu b_{2} x(s)\right] d s \\
& +\frac{\alpha \xi_{1}(t)}{1-\alpha \xi_{1}(\xi)} \int_{0}^{1} G(\xi, s) e(s)\left[\lambda b_{1} x(s)+\mu b_{2} x(s)\right] d s .
\end{aligned}
$$

Thus, we have

$$
\begin{aligned}
(T x)(t) \leq & (\lambda+\mu)\left[b_{1}+b_{2}\right] e(1) \\
& \cdot\left[\int_{0}^{1} G(s, s) x(s) d s+\frac{\alpha \xi_{1}(1)}{1-\alpha \xi_{1}(\xi)} \int_{0}^{1} G(\xi, s) x(s) d s\right] .
\end{aligned}
$$

So, we have

$$
\begin{aligned}
\|(T x)(t)\| \leq & (\lambda+\mu)\left[b_{1}+b_{2}\right] e(1) \\
& \cdot\left[\int_{0}^{1} G(s, s) d s+\frac{\alpha \xi_{1}(1)}{1-\alpha \xi_{1}(\xi)} \int_{0}^{1} G(\xi, s) d s\right]\|x\| .
\end{aligned}
$$

Then, we can have by the definition of $b_{1}, b_{2}$

$$
\|T x\| \leq\|x\|, \forall x \in K \cap \partial \Omega_{2} .
$$

Then, similar to the proof of heorem 8 , we have that result of heorem 9 by Theorem 1 .

Theorem 10. Suppose condition (C1), condition (C2), and condition (C5) hold. Then, for sufficiently small $\lambda, \mu$, problem (2) has at least two positive solutions.

Proof. Firstly, by Lemma 7, there exists constant $\tau>0$ which satisfies

$$
G(\tau) \leq K_{2} \tau
$$

Therefore, setting $\Omega_{1}=\{x \in E:\|x\|<\tau\}$, for any $x \in P$ $\cap \partial \Omega_{1}, t \in[0,1]$, by the above discussion, for the quite small $\lambda, \mu$, we have

$$
\left[\lambda H_{\tau}+\mu Y(\tau)\right] e(1)\left(\int_{0}^{1} G(s, s) d s+\frac{\alpha \xi_{1}(1)}{1-\alpha \xi_{1}(\xi)} \int_{0}^{1} G(\xi, s) d s\right) \leq \tau
$$


We have

$$
\begin{aligned}
(T x)(t) \leq & {\left[\lambda H_{\tau}+\mu Y(\tau)\right] } \\
& \cdot\left[\int_{0}^{1} G(t, s) e(s) d s+\frac{\alpha \xi_{1}(1)}{1-\alpha \xi_{1}(\xi)} \int_{0}^{1} G(\xi, s) e(s) d s\right] \\
\leq & {\left[\lambda H_{\tau}+\mu Y(\tau)\right] e(1) } \\
& \cdot\left(\int_{0}^{1} G(s, s) d s+\frac{\alpha \xi_{1}(1)}{1-\alpha \xi_{1}(\xi)} \int_{0}^{1} G(\xi, s) d s\right) \\
\leq \tau & =\|x\| .
\end{aligned}
$$

Then, we have

$$
\|T x\| \leq\|x\|, \quad \forall x \in P \cap \partial \Omega_{1}
$$

Secondly, by (C5), there exists a constant $l_{3}>1$, which satisfies

$$
a(t, x) \geq K_{1} x, \quad \forall x \geq l_{3}
$$

Letting $r=\max \left\{2 M\|B\|,\left(2 l_{3} / \varepsilon\right), 2 \tau\right\}$, and $\Omega_{2}=\{x \in E$ : $\|x\|<r\}$, for any $x \in P \cap \partial \Omega_{2}, t \in[0,1]$, we have

$x(t)-w(t) \geq x(t)-M\|B\| z(t) \geq x(t)-\frac{M\|B\|}{r} x(t) \geq \frac{1}{2} x(t)$.

Then, $x(t)-w(t) \geq(1 / 2) x(t) \geq(\|x\| / 2) z(t) \geq(r \varepsilon / 2) \geq l_{3}$.

Therefore, by the definitions of $\bar{H}, \bar{Y}$ and the above discussion, we have

$$
\begin{aligned}
(T x)(t) & \geq \int_{0}^{1} G(t, s) e(s)[\lambda \bar{H}(s, x-w)+\mu \bar{Y}(s, x-w)] d s \\
& \geq \int_{0}^{1} G(t, s) e(s) \lambda K_{1}(x-w) d s \\
& \geq \frac{K_{1}}{2} \lambda \min _{0 \leq t \leq 1} \int_{0}^{1} G(t, s) e(s) z(s) d s \geq r=\|x\| .
\end{aligned}
$$

Thus, we have

$$
\|T x\| \geq\|x\|, \forall x \in P \cap \partial \Omega_{2}
$$

Finally, letting

$$
\begin{aligned}
R= & \max \left\{[ \lambda H _ { R } + \mu Y _ { R } ] \left(\int_{0}^{1} G(s, s) e(s) d s+\frac{\alpha \xi_{1}(1)}{1-\alpha \xi_{1}(\xi)}\right.\right. \\
& \left.\left.\cdot \int_{0}^{1} G(\xi, s) e(s) d s\right], 2 r\right\}
\end{aligned}
$$

then, $\tau<r<R$. Set $\Omega_{3}=\{x \in E:\|x\|<R\}$, for any $x \in P \cap \partial$ $\Omega_{3}, t \in[0,1]$, by the definition of operator $T$, we have

$$
\begin{aligned}
(T x)(t) \leq & {\left[\lambda H_{R}+\mu Y_{R}\right] } \\
& \cdot\left(\int_{0}^{1} G(s, s) e(s) d s+\frac{\alpha \xi_{1}(1)}{1-\alpha \xi_{1}(\xi)} \int_{0}^{1} G(\xi, s) e(s) d s\right] .
\end{aligned}
$$

Thus, we have

$$
\|T x\| \leq\|x\|, \quad \forall x \in P \cap \partial \Omega_{3} .
$$

Then, similar to the proof of heorem 8 , we have the result of heorem 10 by Theorem 2 .

Remark 11. The results of these three theorems in our paper also hold under the condition in which nonlinear $a(t, x)$, $b(t, x)$ are both lower semicontinuous.

Remark 12. We can obtain the results of Theorem 10 if we replace condition (C5) with (C6) $K_{1} \leq b_{\infty}^{-} \leq \infty, 0 \leq a_{\infty}^{+} \leq K_{2}$.

\section{Data Availability}

Data sharing is not applicable to this article as no datasets were generated or analyzed during the current study.

\section{Conflicts of Interest}

The authors declare that there is no conflict of interest regarding the publication of this paper.

\section{Authors' Contributions}

All authors contributed equally to the manuscript, and all authors typed, read, and approved the final manuscript.

\section{Acknowledgments}

The authors were supported by the Project of Shandong Province Higher Educational Science and Technology Program (J16LI01).

\section{References}

[1] M. Moshinsky, "Sobre los problems de condiciones a la frontiera en una dimension de caracteristicas discontinuas," Boletin De La Sociedad Matematica Mexicana, vol. 7, pp. 1-25, 1950.

[2] S. Timoshenko, Theory of Elastic Stability, McGraw Hill, New York, 1961.

[3] V. A. Il'in and E. I. Moviseev, "Nonlocal boundary value problem of the second kind for a Sturm-Liouville operator," Differential Equations, vol. 23, pp. 979-987, 1987.

[4] D. Guo and V. Lakshmikantham, Nonlinear Problems in Abstract Cone, Academic Press, Sandiego, 1988.

[5] C. P. Gupta, "Solvability of a three-point nonlinear boundary value problem for a second order ordinary differential 
equation," Journal of Mathematical Analysis and Applications, vol. 168, no. 2, pp. 540-551, 1992.

[6] C. P. Gupta, "A generalized multi-point boundary value problem for second order ordinary differential equations," Applied Mathematics and Computation, vol. 89, pp. 133-146, 1998.

[7] R. Y. Ma and H. Y. Wang, "Positive solutions for a nonlinear three-point boundary value problems," Journal of Mathematical Analysis and Applications, vol. 279, pp. 216-227, 2003.

[8] N. Liu and Y. Liu, "New multi-soliton solutions of a (3+1)dimensional nonlinear evolution equation," Computers \& Mathematics with Applications, vol. 71, no. 8, pp. 1645-1654, 2016.

[9] R. Ma, "Positive solutions for semipositone $(k, n-k)$ conjugate boundary value problems," Journal of Mathematical Analysis and Applications, vol. 252, pp. 220-229, 2000.

[10] T. Qi, Y. Liu, and Y. Zou, "Existence result for a class of coupled fractional differential systems with integral boundary value conditions," The Journal of Nonlinear Sciences and Applications, vol. 10, no. 7, pp. 4034-4045, 2017.

[11] X. Yang, "Green's function and positive solutions for higherorder ODE," Applied Mathematics and Computation, vol. 136, no. 2-3, pp. 379-393, 2003.

[12] D. Jiang, "Positive solutions to singular $(k, n-k)$ conjugate boundary value problems," Acta Mathematica Sinica, vol. 3, pp. 541-548, 2001.

[13] Y. Liu, "Positive solutions using bifurcation techniques for boundary value problems of fractional differential equations," Abstract and Applied Analysis, vol. 2013, Article ID 162418, 7 pages, 2013.

[14] M. Zhong and X. Zhang, "Positive solutions of singularly perturbed $(k, n-k)$ conjugate boundary value problems," Acta Mathematica Scientia (A) China Education, vol. 31, pp. 263272, 2011

[15] R. P. Agarwal, S. R. Grace, and D. O'Regan, "Semipositone higher-order differential equations," Applied Mathematics Letters, vol. 17, no. 2, pp. 201-207, 2004.

[16] H. Su and Z. Wei, "Positive solutions to semipositone $(k, n-k)$ conjugate eigenvalue problems," Nonlinear Analysis: Theory, Methods \& Applications, vol. 69, no. 9, pp. 3190-3201, 2008.

[17] Y. Wang, Y. Liu, and Y. Cui, "Multiple sign-changing solutions for nonlinear fractional Kirchhoff equations," Boundary Value Problems, vol. 2018, no. 1, 2018.

[18] X. Zhang, L. Liu, Y. Wu, and Y. Cui, "A sufficient and necessary condition of existence of blow-up radial solutions for a $k$ -Hessian equation with a nonlinear operator," Nonlinear Analysis: Modelling and Control, vol. 25, pp. 126-143, 2020.

[19] H. Su and X. Wang, "Positive solutions to singular semipositone m-point n-order boundary value problems," Journal of Applied Mathematics and Computing, vol. 36, no. 1-2, pp. 187-200, 2011.

[20] B. Liu and Y. Liu, "Positive solutions of a two-point boundary value problem for singular fractional differential equations in Banach space," Journal of Function Spaces and Applications, vol. 2013, article 585639, pp. 1-9, 2013.

[21] Y. Wang, Y. Liu, and Y. Cui, "Multiple solutions for a nonlinear fractional boundary value problem via critical point theory," Journal of Function Spaces and Applications, vol. 2017, article 8548975, 2017.

[22] Y. Liu and D. O'Regan, "Controllability of impulsive functional differential systems with nonlocal conditions," Elec- tronic Journal of Differential Equations, vol. 194, pp. 1-10, 2013.

[23] H. Yu and Y. Zhan, "Large time behavior of solutions to multidimensional bipolar hydrodynamic model of semiconductors with vacuum," Journal of Mathematical Analysis and Applications, vol. 438, no. 2, pp. 856-874, 2016.

[24] Y. Liu, Y. Zheng, H. Li, F. E. Alsaadi, and B. Ahmad, "Control design for output tracking of delayed Boolean control networks," Journal of Computational and Applied Mathematics, vol. 327, pp. 188-195, 2018.

[25] J. Li and H. Yu, "Large time behavior of solutions to a bipolar hydrodynamic model with big data and vacuum," Nonlinear Analysis-Real World Applications, vol. 34, pp. 446-458, 2017. 